\title{
Local positioning with sensor-enabled passive multistandard RFID transponders
}

\author{
J. Heidrich ${ }^{1}$, D. Brenk ${ }^{1}$, J. Essel ${ }^{1}$, S. Schwarzer ${ }^{2}$, R. Herschmann ${ }^{3}$, B. Geck ${ }^{3}$, G. Fischer ${ }^{1}$, and R. Weigel ${ }^{1}$ \\ ${ }^{1}$ Institute for Electronics Engineering, University of Erlangen-Nuremberg, Erlangen, Germany \\ ${ }^{2}$ Siemens AG Corporate Technology, Munich, Germany \\ ${ }^{3}$ Institute of Radiofrequency and Microwave Engineering, Leibniz Universität Hannover, Germany
}

\begin{abstract}
RFID is used today in many fields of every day life like access control, anti-theft protection or logistics. Within this article a short overview of the basic RFID principles and the EPC protocol flow is given at first. Afterwards new design approaches for RFID systems within the scope of the research project RFID-S are presented.
\end{abstract}

\section{Basic principles of RFID}

There are two basic physical RFID principles. Figure 1 shows the near-field RFID system which operates at $125 \mathrm{kHz}$ and $13.56 \mathrm{MHz}$. It is based on the transformer principle with the boundary condition that the respective coils of the reader and the transponder antenna are only loosely coupled over the air interface (EPCglobal, 2006). For passive systems the RF signal of the reader transmits both information and energy to the transponder. Figure 2 illustrates the backscatter principle which is based on propagation of electromagnetic waves in the far-field. It is typically used at UHF frequencies from $433 \mathrm{MHz}$ to $2.45 \mathrm{GHz}$. The reader transmits data to the tag with a modulated RF signal. After that the reader can receive data from the tag by transmitting an unmodulated RF signal to the tag. The tag responds to this by modulating the reflection coefficient of its antenna, which in turn modulates the transmitted signal of the reader. Therefore, information is backscattered to the reader (EPCglobal, 2007). In general the transponder chip for both methods consists of a modulator, a rectifier and several other components which here are represented by a nonlinear load connected in parallel (Curti et al., 2007; Finkenzeller).

Figure 3 shows the steps by which a reader inventories and accesses a single tag within the EPC protocol (EPCglobal,

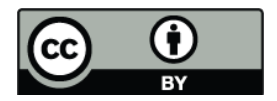

Correspondence to: J. Heidrich (heidrich@ieee.org)
2007). At the beginning a reader selects a certain amount of transponders within its operating range with one or more $\mathrm{Se}$ lect commands. Then the so-called inventory round begins. The tags receive a Query command. All tags which match the select parameters load a random number into their respective slot counter in the digital part. If this value is zero the related tag responds to the reader with a 16 bit random number. Otherwise there would be no reply. The reader sends further QueryRep commands to the selected tags and every time they decrement their slot counter until all have reached zero values. When the reader recognizes the response of the tag then it sends an acknowledge back to the tag with the same random number. Now there would be two possible outcomes. If the received random number is valid the tag responds again. It sends the protocol control word, which contains physicallayer information, for example the length of the EPC or if user memory is implemented in the chip. At last it sends its Electronic Product Code (EPC). The EPC is a code that identifies the object to which a tag is affixed. If the random number was not valid the transponder does not respond. After that the reader sends a $R e q \_R N$ (Request Random number) command to the tag with the current $R N 16$. If the value is valid, the tag generates a new 16 bit random number which is also called handle and sends it to the reader. Every time the reader or this tag sends a message, this handle should be used as an identifier.

\section{Research project RFID-S}

The research project RFID-S deals with passive and locatable multistandard CMOS transponders with sensor functionality. It is supported by the Bavarian Research Foundation (Bayerische Forschungsstiftung, 2007). A purely passive RFID tag is developed which supports the EPC protocols both for HF and UHF in the European area $(13.56 \mathrm{MHz}$ and $868 \mathrm{MHz}$ ). Besides the standard RFID applications further

Published by Copernicus Publications on behalf of the URSI Landesausschuss in der Bundesrepublik Deutschland e.V. 

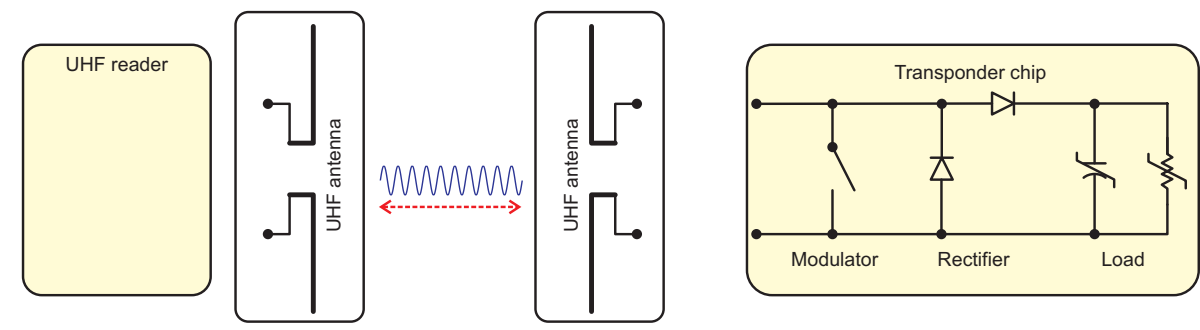

Fig. 1. Inductive field coupling.
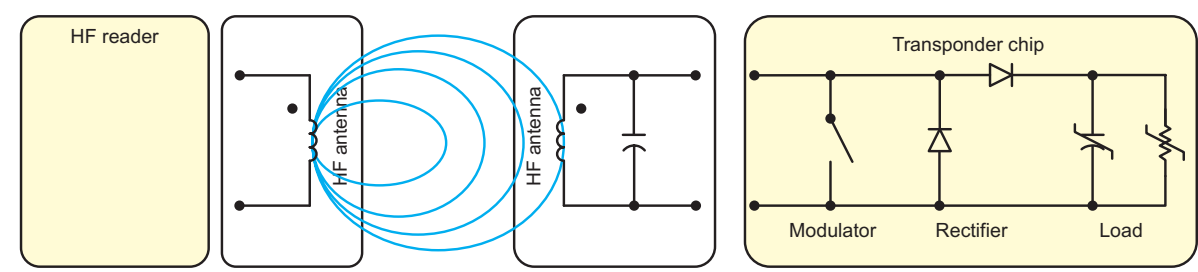

Fig. 2. Backscatter principle.

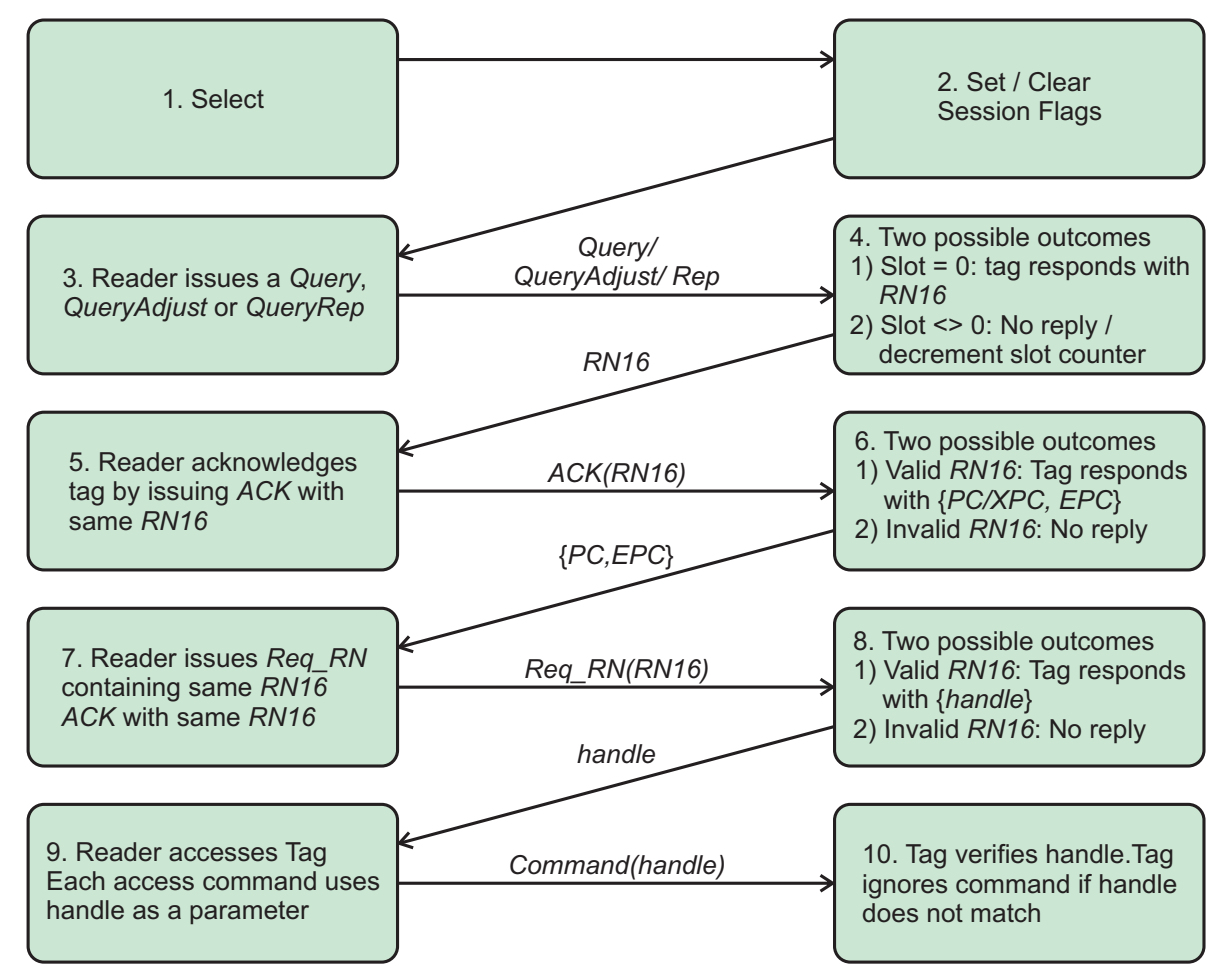

Fig. 3. Communication within the EPC protocol (EPCglobal, 2007).

features are implemented: a radar function is used to measure the distance between the reader and a tag. The second one is a sensor functionality. Therefore, it is possible to acquire sensing data at the location of the transponder, for example the current temperature. The Institute for Electronics Engineering at the University of Erlangen-Nuremberg is responsible for the chip design of the RFID transponder, the
Institute of Radiofrequency and Microwave Engineering at the Leibniz Universität Hannover cares of the antenna design. Siemens AG Corporate Technology in Munich, Germany, develops the reader hardware and the related software to control it. The designed transponder chips are processed in the fabs of Infineon. The actual developments within the scope of this project are described in Brenk (2009); Brenk 


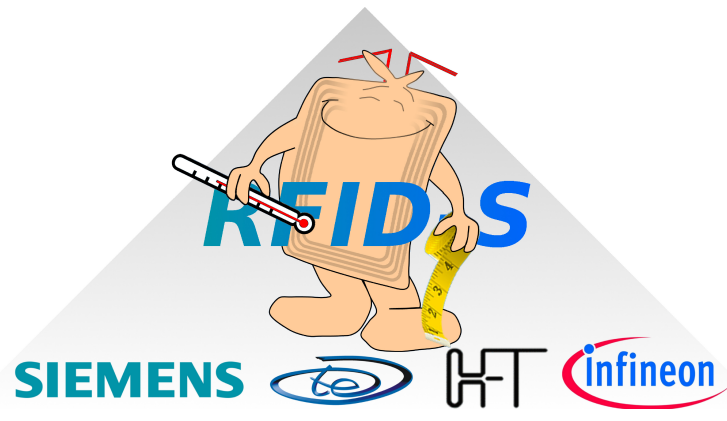

Fig. 4. Research project RFID-S.

et al. (2009a,b,c); Essel et al. (2009a,b,c); Heidrich et al. (2009a,b); Essel et al. (2010a); Heidrich et al. (2010a); Brenk et al. (2010a,b); Essel et al. (2010b); Heidrich et al. (2010b); Meyer et al. (2010a); Heidrich et al. (2010c,d,e); Meyer et al. (2010b); Essel et al. (2010c); Brenk et al. (2010c); Essel et al. (2010d); Heidrich et al. (2010f).

Figure 5 demonstrates the clock generation for $\mathrm{HF}$ and UHF systems. Using near-field systems the reference clock of the transponder is derived from the RF signal of the related reader (EPCglobal, 2006). Here the frequency is $13.56 \mathrm{MHz}$, for example. Because of this relatively low value it can be recovered. Then it can be used directly or can be adjusted easily using clock dividers in the digital part of the tag. At UHF the possible RF frequency is between $860 \mathrm{MHz}$ to $960 \mathrm{MHz}$ according to the EPC protocol for UHF (EPCglobal, 2007). Using this value directly or adapt it with related clock dividers would consume a huge amount of power which is not available for passive RFID tags. To overcome this problem a separate oscillator in the transponder chip is used. Regarding the multistandard design within this project the RF signal of the HF reader is used for communication between transponder and HF reader. During data transfer between UHF reader and transponder the clock source for the tag is switched to the internal oscillator to be compliant to the EPC protocol at UHF.

Figure 6 shows schematically all components of the RFID system. As a first design approach separate RFID readers are used for the HF and the UHF range. For the positioning functionality a separate FMCW reader is used which is developed by Siemens. All readers should be combined via a single interface. Therefore, it is possible to control the whole reader system via a common PC application which is independent of the actual used reader hardware.

The whole transponder is placed on a PCB board with standard creditcard size (ID-1). For this demonstrator system the structure of the tag antenna is layouted on the card. It is connected with the packaged chip which contains all necessary transponder components: first there is the analog part for data recovery from the reader, data transmission back to the reader and power supply. The digital part contains the protocol stack. In this system the digital part implements both the standard EPC protocol and additionally two custom commands according to the protocol (EPCglobal, 2007) which control the positioning and sensor features. Moreover, there are the sensor interface including the sensor, several bias circuits and the local oscillator for the UHF part. The actual architecture of the oscillator is a tradeoff between different design criteria like power consumption, necessary accuracy, available components in the used process (resistors, inductors), chip area and costs.

Figure 7 shows the common air interface of that system in detail. The three readers use a proprietary antenna, respectively. Because of the spatial limitations the transponder must use a combined antenna structure for all frequency ranges. The HF and the UHF reader send both data and energy to the transponder. Therefore the transponder antenna must be matched for these frequencies to allow a sufficient power supply up to a certain distance. For the FMCW application at $2.45 \mathrm{GHz}$ there are contrary requirements. Most of the power of the FMCW signal should be reflected back to the FMCW reader for measuring the distance according to the FMCW radar principle. Ideally the antenna should be matched perfectly to the HF and UHF antenna and should be unmatched at the frequency range of the FMCW signal (here 2.45 GHz).

Besides the desired signal components the communication can be influenced by adjacent UHF channels. The distance measurement can be corrupted by interferences in the same frequency range, for example WLAN or by multipath signals.

Figure 8 shows the design of a multistandard antenna for these purposes. It was developed at the Institute of Radio frequency and Microwave Engineering. On the top there is a parallel LC series resonant circuit for the HF path. The related inductance is built by two coils in series on the left and right. In the middle there is the capacitance which is realized by two parallel striplines. At the bottom the folded dipole antenna for the UHF and the microwave path is arranged. The two parts should be connected to the packaged chip via one or two common feeding points.

The EPC protocol for UHF provides ASK, PSK or both modulation schemes for the data transfer from the transponder to the reader (EPCglobal, 2007). The tag manufacturer can choose it and the reader should support both modulation types. Figure 9 shows the reflection coefficient of the transponder antenna in the Smith chart for these different types assuming a sufficiently linear chip impedance. A purely ASK modulation has two states only on the real axis. A purely PSK changes only the phase of the reflection coefficient. Using both types magnitude and phase of the reflection coefficient are modulated and it can be switched between two arbitrary points in the Smith chart. Because the modulation should be performed by a shunt transistor as illustrated in Fig. 2, the admittance view of the Smith chart was used.

The distance measurement is implemented with an FMCW radar. For the sake of understanding the basic FMCW 


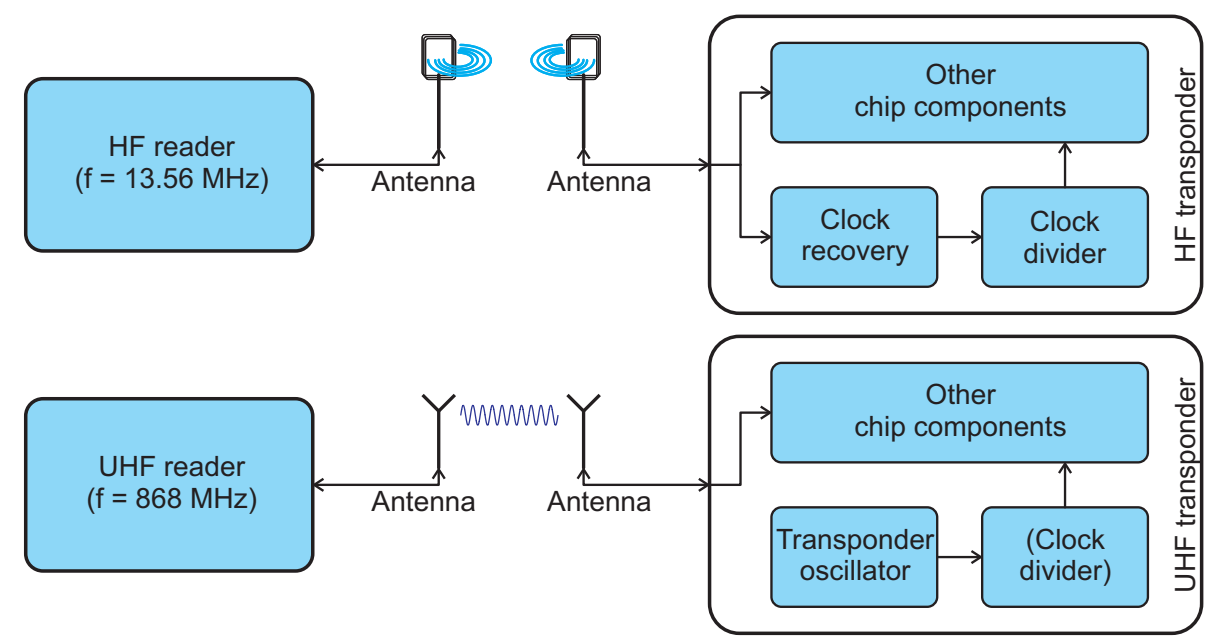

Fig. 5. Generation of the transponder clock.

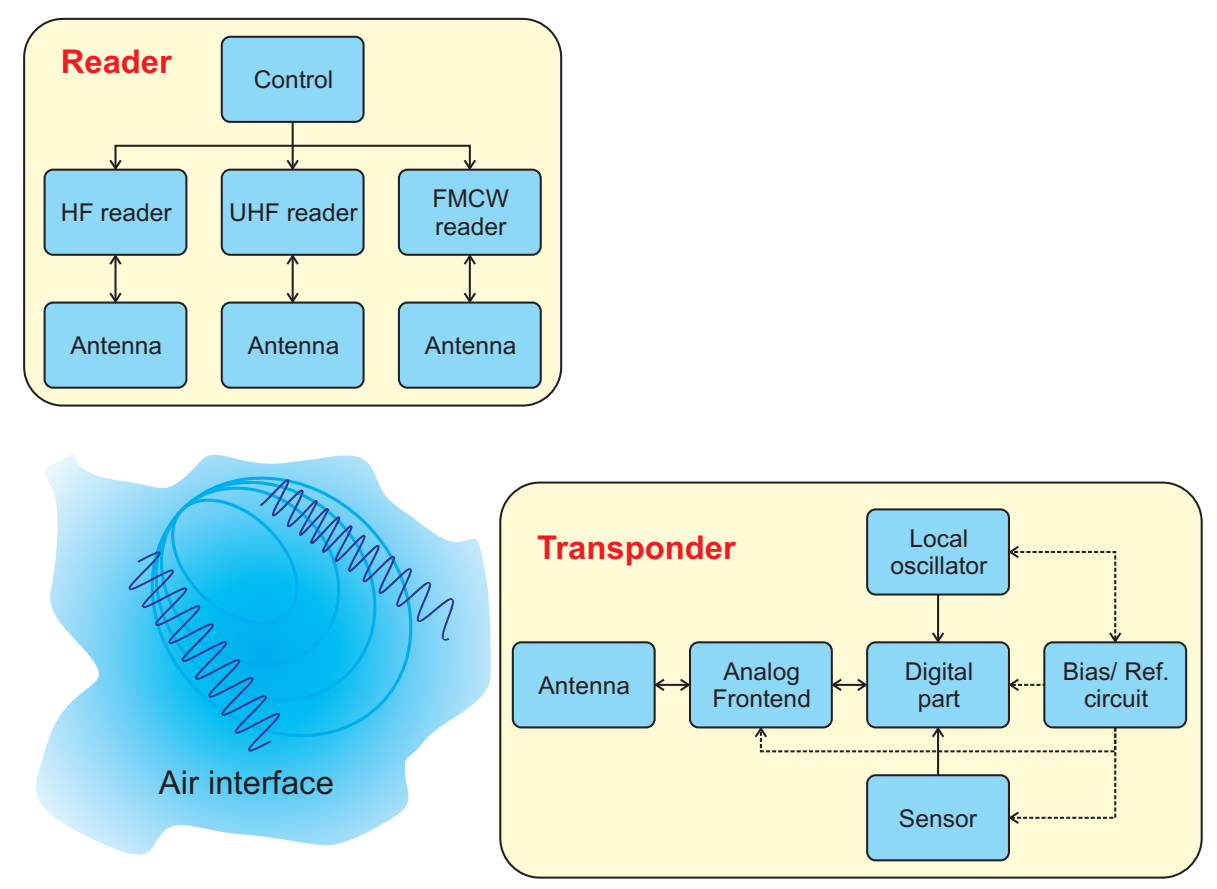

Fig. 6. Block diagram of the whole RFID system.

principle is described briefly. For a deeper insight in the theory of FMCW and radar applications, refer to Barton (2005). Figure 10 shows the block diagram of the FMCW reader used in this project. It was developed by Siemens AG Corporate Technology in Munich, Germany.

The FMCW radar generates and radiates an FMCW signal as shown in Fig. 11a. The ramp generator and the VCO generate the current frequency of the carrier signal within the modulation interval $T / 2$, which increases linearly from $f=f_{0}$ to $f=f_{0}+B$. Therefore, the common FMCW signal can be expressed as
$f(t)=f_{0}+\frac{B}{T} \cdot t$

After transmission, this signal can be reflected at any target within the operating range of the reader and will arrive again at the reader antenna after the common run-time $\tau_{\mathrm{Tr}}$. Figure $11 \mathrm{~b}$ shows the related frequency curves of the backscattered and the transmitted signal. They have similar waveforms and are only displaced by the run-time $\tau_{\mathrm{Tr}}$ from the reader to the target and back. 


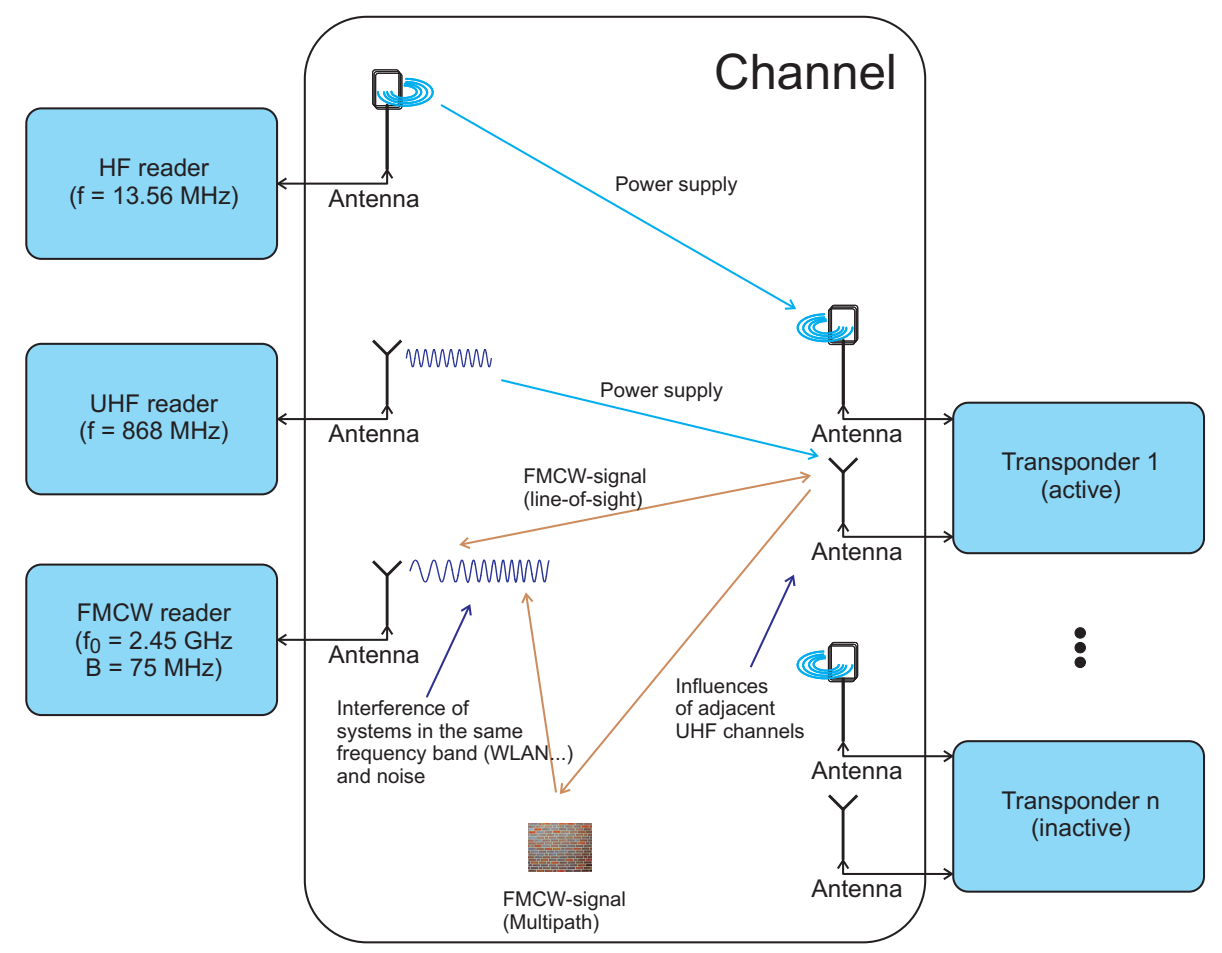

Fig. 7. Common channel of the multistandard system.

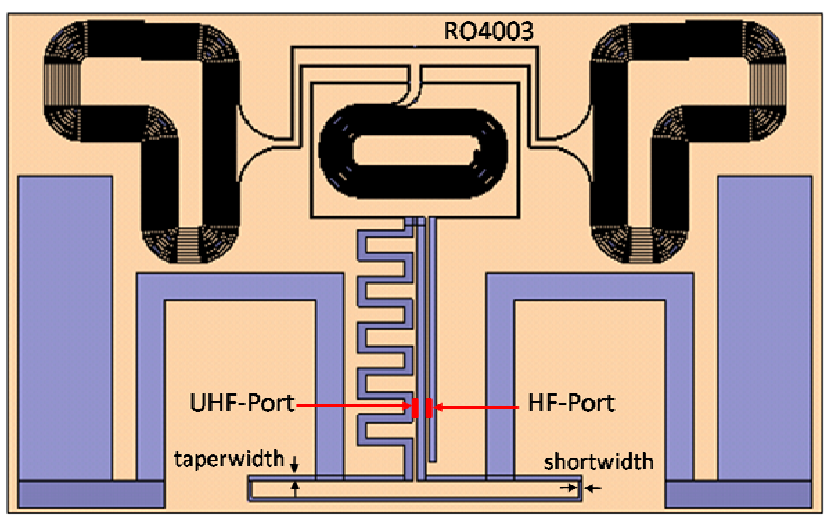

Fig. 8. Design of a multistandard antenna (courtesy of Institute of Radiofrequency and Microwave Engineering).

The run-time causes a characteristic frequency shift $\Delta f$ which is used for the distance measurement (see Eq. 2)

$\frac{B}{T / 2}=\frac{\Delta f}{\tau_{\operatorname{Tr}}} \Leftrightarrow \tau_{\operatorname{Tr}}=\frac{\Delta f}{B} \cdot \frac{T}{2}$,

where $T$ is the modulation interval of the FMCW system and $\mathrm{B}$ is the related bandwidth. Now the distance $d$ is calculated as follows:

$d=0.5 \cdot c_{0} \cdot \tau_{\operatorname{Tr}}=\frac{\Delta f \cdot T \cdot c_{0}}{4 \cdot B}$,

where $c_{0}$ is the speed of light.
As stated by Eq. (4) the resolution $\Delta d$ depends on the used bandwidth $B$. Therefore the ISM band of $2.45 \mathrm{GHz}$ is used for the distance measurement because of the higher allowed bandwidth in comparison to the UHF range given by regulation. According to regulation at this ISM band, a maximum output power of $13 \mathrm{dBm}$ is allowed (ETSI EN 300 440-1, 2001).

$$
\begin{aligned}
\Delta d=\Delta f \cdot \frac{T}{B} \cdot c_{0} \approx \frac{1}{T} & \cdot \frac{T}{B} \cdot c_{0}=\frac{c_{0}}{B} \\
S_{r}(t)=S_{\mathrm{LOS}}(t) \cdot \Gamma(t) & +\sum_{i=0}^{n} S_{M P i}(t) \cdot \Gamma(t) \\
& +\sum_{j=0}^{m} S_{T j}(t) \cdot \Gamma_{T j}(t)
\end{aligned}
$$

Assuming ideal conditions the distance information can be evaluated without modifications. However, in every typical application field, especially indoor environments, other undesired targets within the operating range of the reader can also reflect a certain ratio of the radiated FMCW signal according to their radar cross section (RCS). Their backscattered components superpose with the desired component of the transponder and would falsify the related baseband signal at the output of the reader. Besides the reflection of other objects multipath reflections are also of concern. Figure 12 demonstrates the resulting common channel for the distance measurement with UHF transponders. The different components can be expressed with Eq. (5), where $S_{\mathrm{LOS}}(t)$ is the line-of-sight signal, $S_{\mathrm{MP} i}(t)$ the multipath signals, $S_{T j}(t)$ reflections from other targets and $\Gamma(t)$ and $\Gamma_{T j}(t)$ the related 

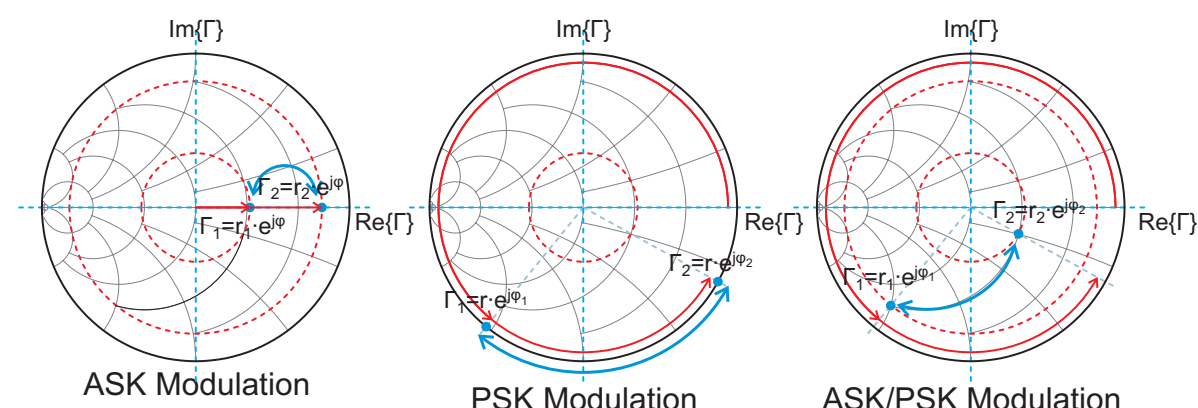

Fig. 9. Possible backscatter modulation schemes.

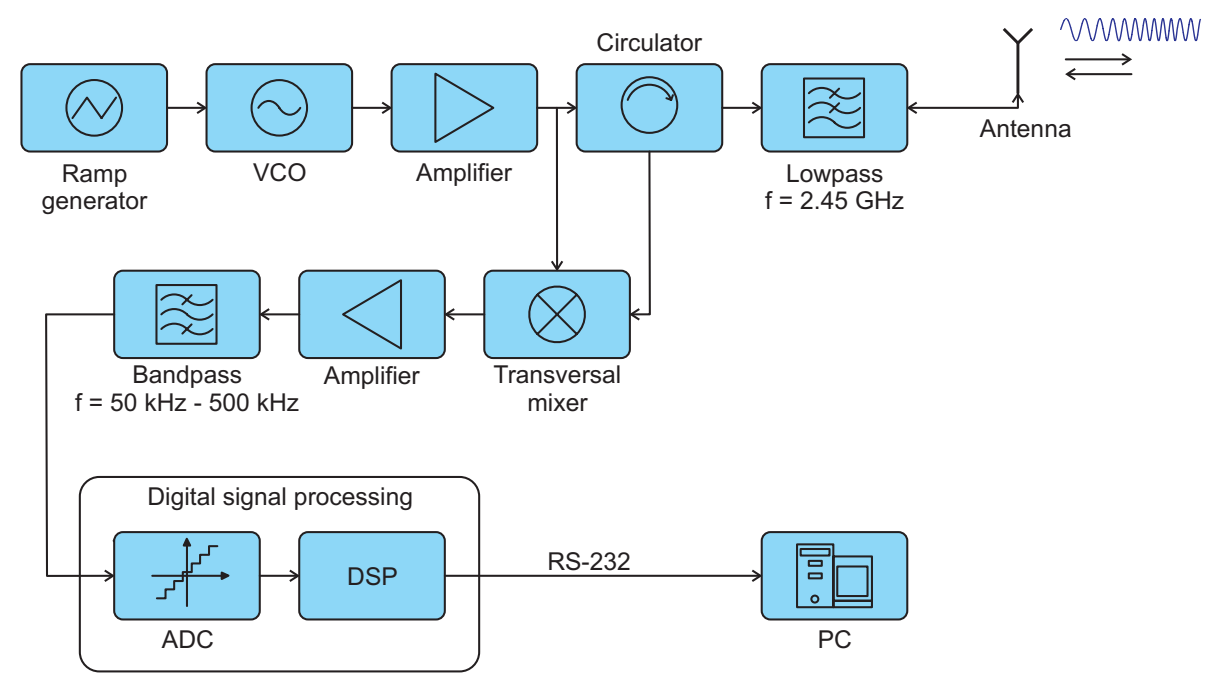

Fig. 10. Block diagram of the FMCW reader.

reflection coefficients. The line-of-sight signal which is directly backscattered from the UHF transponder to the FMCW reader is the desired component for the distance measurement. To distinguish it from the components of other targets the reflection coefficient (RCS in terms of radar theory) of the transponder antenna is modulated. This can be achieved by continuously switching on and off the basic backscatter modulator with a certain modulation signal. This principle is called modulated backscattering (Heidrich et al., 2009a, 2010e; Wiebking, 2003; Vossiek et al., 1999; Heide et al., 2005). It causes a frequency shift of the backscattered signal component from the transponder in the baseband spectrum of the FMCW reader with the used modulation frequency $f_{\text {mod }}$, as still will be shown in Fig. 16 for a simplified setup. However, the undesired multipath components cannot be suppressed with this approach. They are also modulated and therefore frequency shifted. Their influence can only be diminished by reducing the coverage area of the reader antenna.
Figure 13 shows the ideal modulation states for both frequency ranges. In the UHF region the antenna should be matched as good as possible in the unmodulated state which is related to the middle of the Smith chart. In the modulated state the reflection coefficient is within a certain area around the matching point. The actual value is a tradeoff between protocol requirements (80-100\% ASK) (EPCglobal, 2007), detectability and power consumption. For the distance measurement at $2.45 \mathrm{GHz}$ the reflection coefficient should be near the border of the Smith chart for maximal reflection, so a purely PSK modulation would be best.

However, in a real system the modulation is often a mixture of ASK and PSK modulation. To reduce the complexity of the transponder circuit the basic backscatter modulator should be used for both the UHF and the microwave region $(2.45 \mathrm{GHz})$. Here it is realized by a single shunt transistor, so a modulation is performed between the high resistance state and the on resistance of the related transistor. Figure 14 shows the respective curves in the Smith chart for the common case. It must be mentioned that this describes only the common principle with the assumption of a sufficiently high linearization of the input impedance of the transponder which 
a)

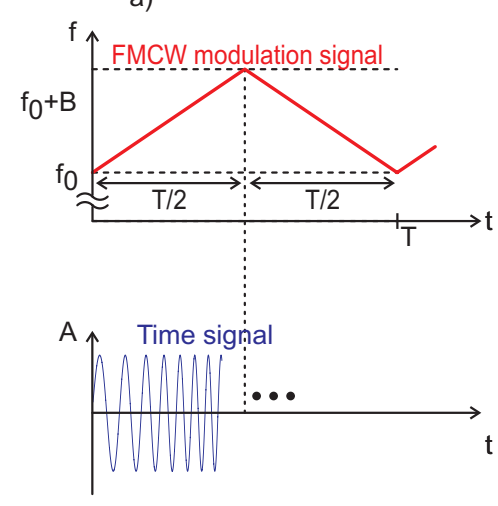

b)

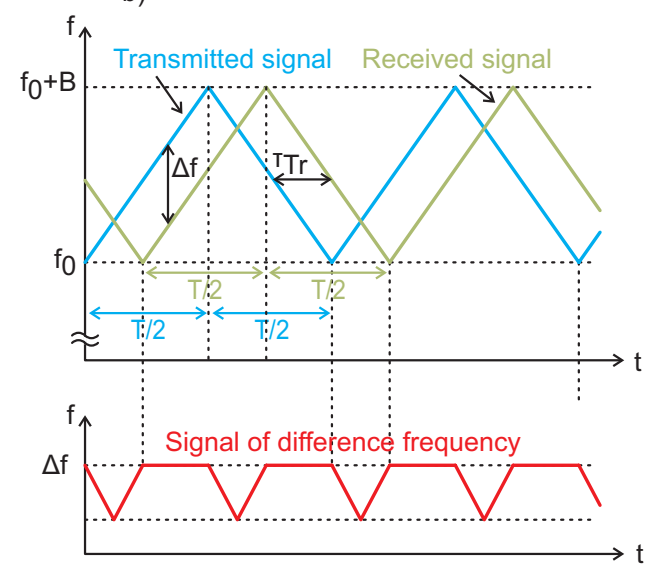

Fig. 11. FMCW signal.

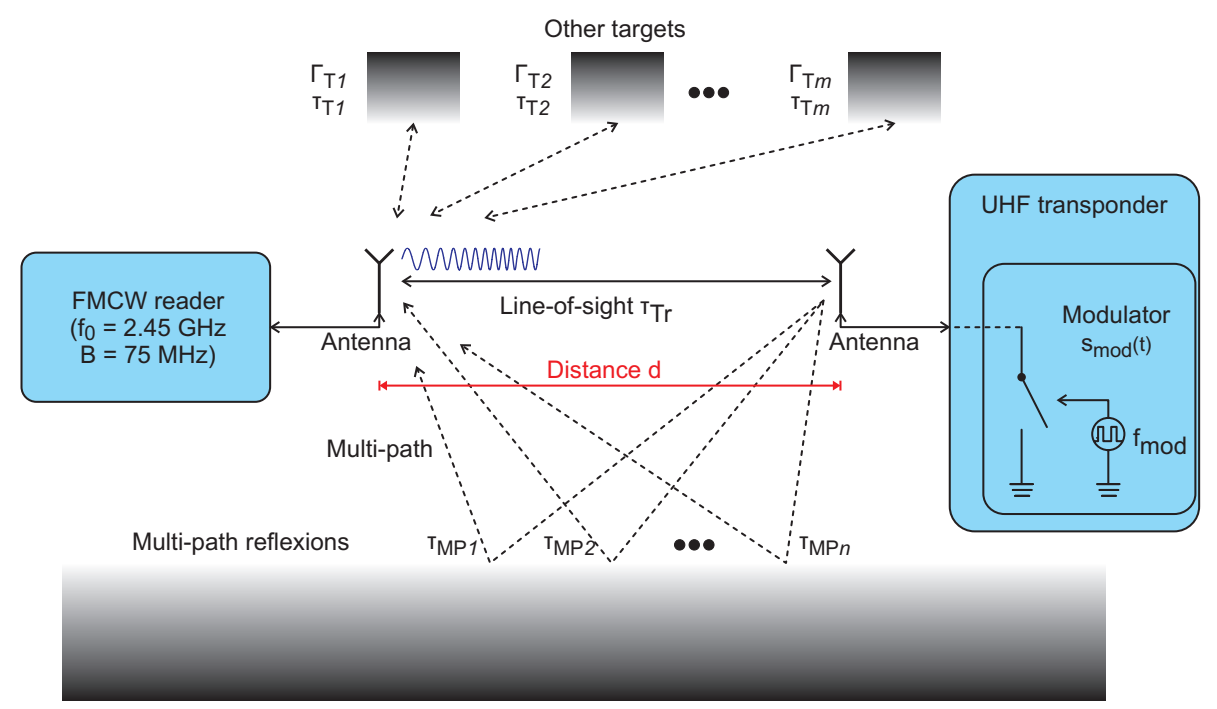

Fig. 12. Distance measurement with UHF transponder.

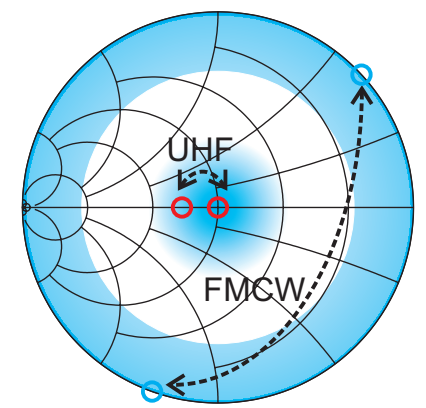

Fig. 13. Ideal modulation states.

is performed with a passive matching network for power matching between antenna and transponder. The matching and the antenna design decide which states in the Smith chart
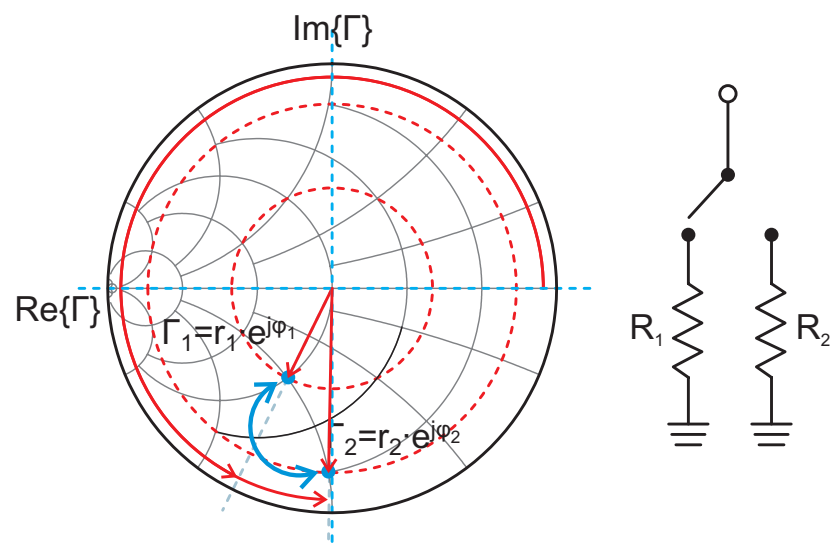

Fig. 14. Possible modulation scheme of the transponder.

Adv. Radio Sci., 8, 263-273, 2010 


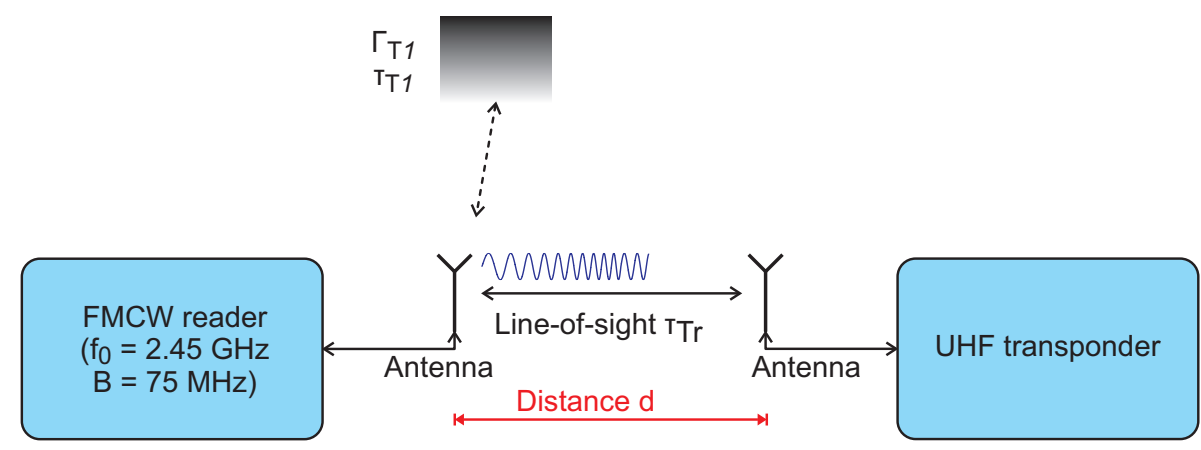

Fig. 15. Simplified channel.

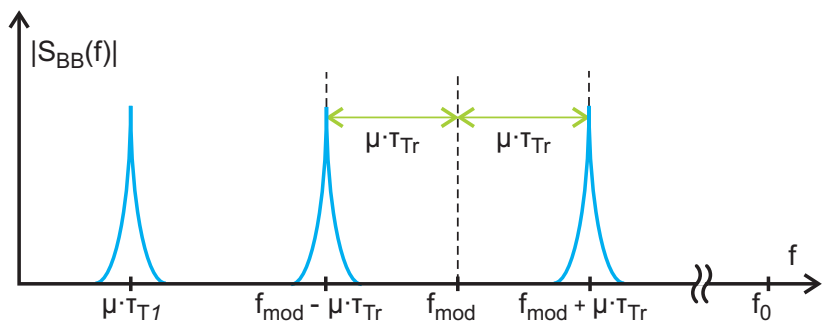

Fig. 16. Baseband spectrum of the FMCW reader.

at the different frequency ranges can be used. As a first design strategy power matching of the antenna at UHF is performed and then the reflection characteristics for the distance measurement will be optimized.

To describe the principle of modulated backscattering Fig. 15 shows the channel of Fig. 12 with several simplifications: a harmonic modulation signal is assumed in the transponder. Only one additional target is within the operating range of the reader and multipath effects are not of concern. Figure 16 shows the resulting baseband signal at the output of the FMCW reader.

The spectral component backscattered from the transponder is segmented in an upper and a lower sideband around the selected modulation frequency $f_{\text {mod }}$, respectively. The respective frequency difference between these two peaks only depends on the run-time $\tau_{\mathrm{Tr}}$ from the reader to the transponder and back. The other parameter $\mu$ is a constant given by the system architecture (Heidrich et al., 2009a). The component of the other target (with the run-time $\tau_{T 1}$ ) is not modulated and therefore not frequency shifted. It can be clearly separated from the signal components of the transponder using a low-pass filter (Heidrich et al., 2009b; Essel et al., 2009c, 2010a, 2009a).

The actual distance value can be evaluated with the following DSP algorithm (see Fig. 17): the baseband signal of the FMCW reader is digitized and its constant component is removed by subtracting its mean value. Then the time signal is conditioned using known DSP techniques like weighting with a window function and zero-padding (Wiebking, 2003;
Smith, 2006). Then the spectrum of this signal is calculated with the FFT algorithm.

Figure 18a illustrates the resulting FFT spectrum according to Fig. 16. Besides the two desired peaks of the backscattered transponder signal, the spectrum is also influenced by noise. Therefore, a certain threshold value is defined. The magnitude of an FFT point must exceed it to be detected as a signal component. Around the detected two maxima the magnitude must also diminish by a certain value. Otherwise every value above the threshold would be regarded as a maximum.

The difference of the related FFT points is calculated and then converted to its equivalent frequency difference, as determined by Eqs. (6) and (7).

$\Delta \mu=\mu_{\text {max right }}-\mu_{\text {max left }}$

$\Delta F=\frac{\Delta \mu \cdot f_{\text {Sample }}}{M}$

$f_{\text {Sample }}$ is the sampling frequency and $M$ the number of FFT points. However, using the FFT algorithm the spectrum is only calculated at equidistant frequency points, as shown in Fig. 18b. This causes discretization errors during the calculation of the frequency difference depending on the distance of the points. Therefore, the detected maximum and its two adjacent FFT points are interpolated with a parabolic curve which approximates the real maximum with high accuracy.

Finally the distance calculation can be performed using Eq. (8)

$d=\frac{\Delta F \cdot T \cdot c_{0}}{4 \cdot B}=\frac{\Delta \mu \cdot f_{\text {Sample }} \cdot T \cdot c_{0}}{4 \cdot M \cdot B}$.

By reducing the distance between FMCW reader and the modulating UHF transponder the two peaks in the baseband spectrum approximate each other more and more. Going below a certain limit the peaks are merging to one and the distance measurement is not possible yet. This value depends on the bandwidth of the two peaks. Figure 19 demonstrates this assuming equal bandwidth and magnitude of the two peaks. Then they merge if they approximate each other more than the $-3 \mathrm{~dB}$ bandwidth. 


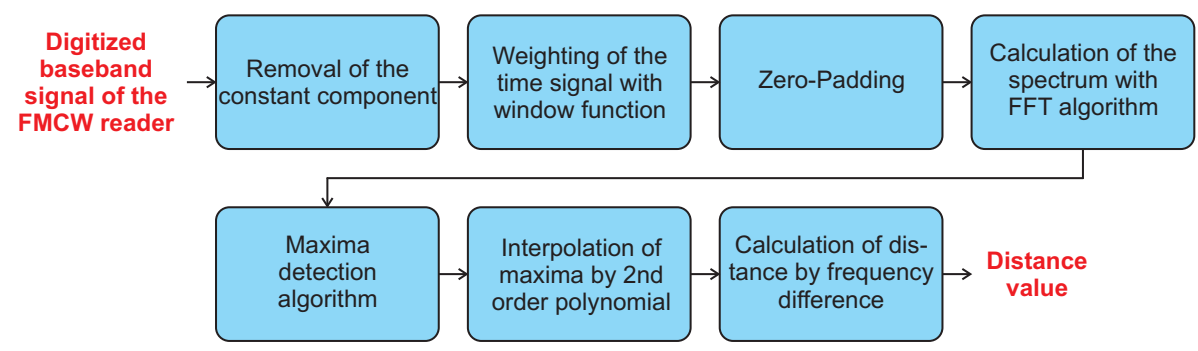

Fig. 17. DSP algorithm for distance calculation.

a)

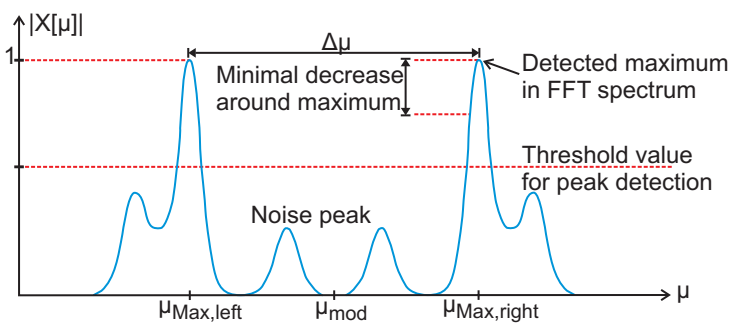

b)

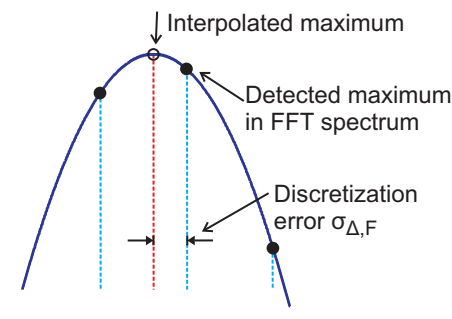

Fig. 18. Processing of FFT spectrum.
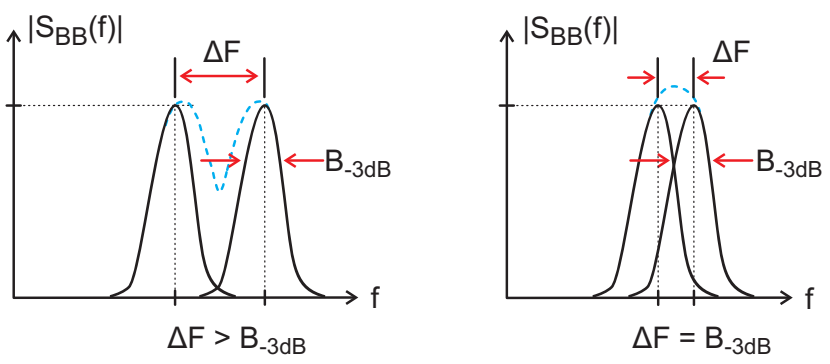

Fig. 19. Resolution of the peaks.

Figure 20 shows schematically the sensor interface of the transponder used in this project. The control of this component is achieved with a defined custom command compliantly to the EPC protocol. When the reader sends the related command this function is activated and the digital part enables a clock signal CLK for an ADC which is used for digitizing of the sensor data. $V_{\text {Ref }}$ is an internal reference voltage for the ADC. Within the scope of this project one of four different sensors should be read by a multiplexer. A temperature sensor and two other sensors which can detect the field strength for HF and UHF in the analog frontend will be implemented in the transponder chip. Optionally, there is a generic interface to connect an external sensor to the chip. The sensor data is digitized by the ADC. The digitized sensor data are sent to the reader via the digital part.

Wireless sensing circuits implemented in passive UHF transponders offer several advantages: in logistics one can survey the current temperature of cargo goods, for example food. Another point is facility surveillance where the hu- midity or luminance in storerooms can be measured. All measurements are performed passively, so there is no need to change batteries after certain time periods. This results also in a higher reliability because of no weak battery voltage and no battery contact problems (Brenk et al., 2009b,c).

Table 1 shows an example how the two custom commands for positioning and sensing can be implemented in the digital part of the transponder. Prescribed by the protocol all custom commands must have the bit sequence "11100000" at first (EPCglobal, 2007). The following four Bits determine the Sensor or Locate functionality $(0 \mathrm{xE} / 0 \mathrm{xF})$. With the first parameter of the Sensor command the desired sensor is selected. Parameter 2 states, if the sensor function should occur one time or continuously. The Locate command enables the distance measurement. The sole parameter chooses the duration $T$ of this measurement. This value depends on the modulation interval of the FMCW reader which influences the resolution of the distance measurement (see Eq. 4, Figs. 11 and 19) (Heidrich et al., 2009a, 2010e; Barton, 2005).

\section{Conclusion}

This article has given a short overview of basic and novel design approaches of RFID systems which are covered by the research project RFID-S. For the actual circuit implementations developed within the project refer to Brenk et al. (2009a); Brenk (2009); Essel et al. (2009a); Brenk et al. (2009b); Essel et al. (2009b); Heidrich et al. (2009a); Brenk et al. (2009c); Heidrich et al. (2009b); Essel et al. (2009c, 2010a); Heidrich et al. (2010a); Brenk et al. (2010a,b); Essel 


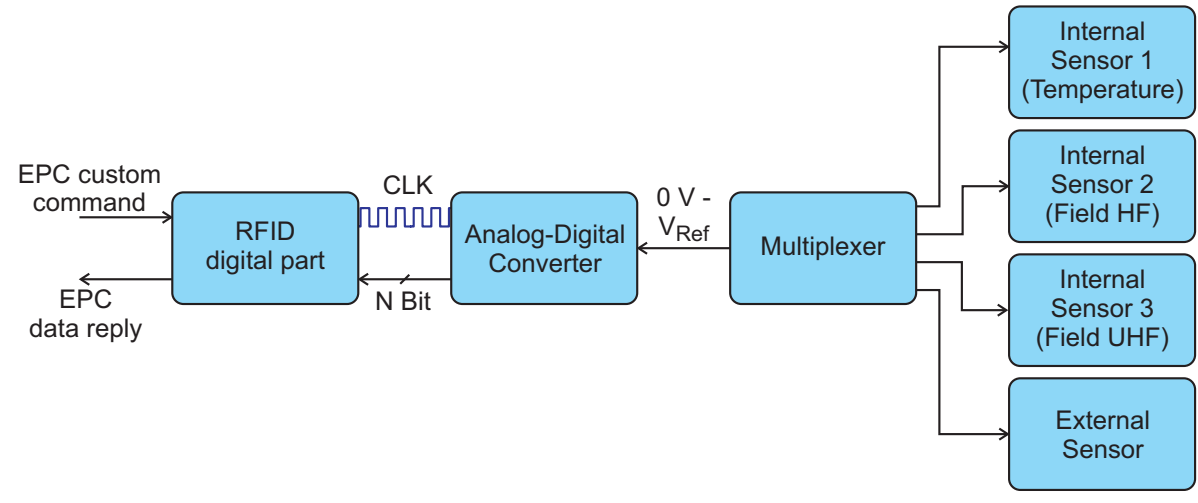

Fig. 20. Sensor interface of the transponder.

Table 1. Implementation of related custom commands.

\begin{tabular}{|c|c|c|c|}
\hline Command & Code & $\begin{array}{l}\text { Parameter } 1 \\
\text { (select source) }\end{array}$ & $\begin{array}{l}\text { Parameter } 2 \\
\text { (select function) }\end{array}$ \\
\hline Sensor & 0xE0EX & $\begin{array}{l}X=0 \mathrm{~b} 00 \mathrm{xx}=\operatorname{src} 1 \\
X=0 \mathrm{~b} 01 \mathrm{xx}=\operatorname{src} 2 \\
X=0 \mathrm{~b} 10 \mathrm{xx}=\operatorname{src} 3 \\
X=0 \mathrm{~b} 11 \mathrm{xx}=\text { reserved } \\
\text { le 0xE0E5: } 111000001\end{array}$ & $\begin{array}{l}X=0 \mathrm{bxx} 00=\text { single } \\
X=0 \mathrm{bxx} 01=\text { continuous } \\
X=0 \mathrm{bxx} 10=\text { reserved } \\
X=0 \mathrm{bxx} 11=\text { reserved } \\
00101\end{array}$ \\
\hline Command & Code & & $\begin{array}{l}\text { neter } 1 \\
\text { eout) }\end{array}$ \\
\hline Locate & $\begin{array}{r}\text { 0xE0FX } \\
\text { Exan }\end{array}$ & $\begin{array}{l}\mathrm{X}=0 \mathrm{~b} 0000 \ldots \text {.. } 0 \mathrm{~b} 1111 \\
16 \text { steps à } 50 \mu \mathrm{s} \text { respecti } \\
\text { with mean value } 0 \mathrm{~b} 1000 \\
\text { ple } 0 \mathrm{xE} 0 \mathrm{FC}: 111000001\end{array}$ & $\begin{array}{l}1.0 \mathrm{~ms}: 0.6 . .1 .4 \mathrm{~ms} \\
11100\end{array}$ \\
\hline
\end{tabular}

et al. (2010b); Heidrich et al. (2010b); Meyer et al. (2010a); Heidrich et al. (2010c,d,e); Meyer et al. (2010b); Essel et al. (2010c); Brenk et al. (2010c); Essel et al. (2010d); Heidrich et al. (2010f).

\section{References}

Barton, D. K.: Radar Systems Analysis and Modeling, Boston, London: Artech House, 2005.

Bayerische Forschungsstiftung, Munich, Germany: Cmos-rfid-s: Verbesserung drahtloser identifikationssysteme, online, available: http://www. forschungsstiftung.de/index.php/Projekte/Details/

CMOSRFID-S-Verbesserung-drahtloser-Identifikationssysteme. html, 2007.

Brenk, D.: Ultra low power sensor-enabled multistandard rfids, in: Department of Materials Science 6 Electrical Engineering Materials University of Erlangen-Nuremberg, editor, Advanced Electronics - Materials, Devices and System Applications, volume Proceedings of the 3rd Chinese-German Summer School in Erlangen, 4-12 May 2009, pp 107-115, Gruner Druck GmbH, Erlangen, 2009.
Brenk, D., Essel, J., Heidrich, J., Cilek, F., and Weigel, R.: An ultra low power adc for sensor applications in passive uhf rfids, in: Proc. Semiconductor Conference Dresden SCD 2009, 29-30 April 2009a.

Brenk, D., Essel, J., Heidrich, J., and Weigel, R.: Ultra low-power techniques for sensor-enhanced rfid tags, in: Proc. IEEE MTT-S International Microwave Workshop on Wireless Sensing, Local Positioning, and RFID IMWS 2009, pp 1-4, 2009 b.

Brenk, D., Essel, J., Heidrich, J., Weigel, R., Hofer, G., and Holweg, G.: Wireless sensing by means of passive multistandard rfid tags, in: Proc. IEEE Sensors, pp 61-64, 25-28 October 2009c.

Brenk, D., Heidrich, J., Eßel, J., Fischer, G., and Weigel, R.: Sensor-enhanced passive cmos-rfid-tags, tm - Technisches Messen, 7-8, 404-412, 2010a.

Brenk, D., Essel, J., Heidrich, J., and Weigel, R.: Advances in Wireless Sensors and Sensor Networks, chapter Locatable, sensorenabled multistandard RFID tags, pp 125-150, Lecture Notes in Electrical Engineering, Springer Berlin Heidelberg, 2010b.

Brenk, D., Essel, J., Heidrich, J., Weigel, R., Hofer, G., and Holweg, G.: Sensor data acquisition with a 79-fj/conversion-step 7.2-enob successive approximation adc for low-power wireless applications, in: Proc. IEEE Sensors, published, 2010c.

Curti, J.-P., Declercq, M., Dehollain, C., and Joehl, N.: Design and 
optimization of passive UHF RFID systems, Springer, New York, 2007.

EPCglobal: Radio-frequency identity protocols - hf version $2 \mathrm{rfid}-$ draft version 0.0.9, EPCglobal Standard, available: http://www. epcglobalinc.org/standards/hfg2/, July 2006.

EPCglobal: Radio-frequency identity protocols - class-1 generation-2 rfid v1.2.0, EPCglobal Standard, EPCglobal Inc., available: http://www.epcglobalinc.org/standards/uhfc1g2, March 2007.

Essel, J., Brenk, D., and Weigel, R.: Rfid and wireless sensing, in: SENSOR+TEST Conference 2009 - SENSOR 2009 Proceedings II, pp 167-172, 26-28 May 2009a.

Essel, J., Brenk, D., Heidrich, J., and Weigel, R.: A highly efficient uhf rfid frontend approach, in: Proc. IEEE MTT-S International Microwave Workshop on Wireless Sensing, Local Positioning, and RFID IMWS 2009, pp 1-4, 2009 b.

Essel, J., Brenk, D., Heidrich, J., Reinisch, H., Hofer, G., Holweg, G., and Weigel, R.: Highly efficient multistandard rfids enabling passive wireless sensing, in: Proc. Asia Pacific Microwave Conf, APMC 2009, pp 2228-2231, December 2009c.

Essel, J., Brenk, D., Heidrich, J., and Weigel, R.: Analog frontend for passive multi-standard rfid transponders, in: Proc. Third EURASIP Workshop on RFID Technology, pp 47-50, 2010a.

Essel, J., Brenk, D., Heidrich, J., Fischer, G., and Weigel, R.: Passive cmos rfids: Developments towards sensing and ranging, in: Sensoren und Messsysteme 2010, pp 501-506, 18-19 May 2010b.

Essel, J., Brenk, D., Heidrich, J., Weigel, R., Hofer, G., and Holweg, G.: A low-power multi-standard frontend for wireless sensor networks, in: Proc. IEEE Sensors, published, 2010c.

Essel, J., Brenk, D., Heidrich, J., Holweg, G., and Weigel, R.: Design of a highly efficient cmos rectifier for passive communication systems, in: Proc. Asia Pacific Microwave Conf. APMC 2010, in press, 2010d.

ETSI EN 300 440-1: Electromagnetic compatibility and radio spectrum matters (erm); short range devices; radio equipment to be used in the $1 \mathrm{ghz}$ to $40 \mathrm{ghz}$ frequency range; part 1: Technical characteristics and test methods, ETSI EN 300 440-1, September 2001.

Finkenzeller, K.: RFID Handbook: Fundamentals and Applications in Contactless Smart Cards, Radio Frequency Identification and Near-Field Communication, Wiley, 2010.

Heide, P., Ilg, J., Roskosch, R., Hofbeck, K., Piesch, W., and Vossiek, M.: Patent us000006946949b2, September 2005.

Heidrich, J., Brenk, D., Essel, J., Fischer, G., Weigel, R., and Schwarzer, S.: Local positioning with passive uhf rfid transponders, in: Proc. IEEE MTT-S International Microwave Workshop on Wireless Sensing, Local Positioning, and RFID IMWS 2009, pp 1-4, 2009a.
Heidrich, J., Brenk, D., Essel, J., Hofer, G., Holweg, G., Fischer, G., and Weigel, R.: Low voltage reference cells for uhf transponders with advanced features, in: Microwaves, Communications, Antennas and Electronics Systems, 2009, COMCAS 2009, IEEE International Conference on, pp 1-8, November 2009b.

Heidrich, J., Brenk, D., Essel, J., Schwarzer, S., Seemann, K., Fischer, G., and Weigel, R.: The roots, rules, and rise of rfid, Microwave Magazine, IEEE, 11(3), 78-86, 2010a.

Heidrich, J., Brenk, D., Essel, J., Heinrich, M., Hofer, G., Holweg, G., Fischer, G., and Weigel, R.: Design of a low-voltage reference circuit with reconfigurable temperature range for rfid applications, in: Microwave Symposium Digest (MTT), 2010 IEEE MTT-S International, pp 1516-1519, May 2010b.

Heidrich, J., Brenk, D., Essel, J., Heinrich, M., Hofer, G., Holweg, G., Weigel, R., and Fischer, G.: Design of an electronically adaptable low-power reference cell for low-cost cmos processes, in: Microwave Radar and Wireless Communications (MIKON), 2010 18th International Conference on, pp. 1-4, June 2010c.

Heidrich, J., Brenk, D., Essel, J., Heinrich, M., Jung, M., Hofer, G., Holweg, G., Weigel, R., and Fischer, G.: Design of a lowpower voltage regulator for rfid applications, in: Computational Technologies in Electrical and Electronics Engineering (SIBIRCON), 2010 IEEE Region 8 International Conference on, pp. 552-557, July 2010d.

Heidrich, J., Brenk, D., Essel, J., Schwarzer, S., Weigel, R., and Fischer, G.: Distance measurement with uhf transponders by means of modulated backscattering (invited paper), in: International Conference on Applied Electromagnetics and Communications ICECOM 2010, pp 1-4, September 2010e.

Heidrich, J., Brenk, D., Essel, J., Heinrich, M., Hofer, G., Holweg, G., Schwarzer, S., Meyer, J., Herschmann, R., and Geck, B., Weigel, R., and Fischer, G.: Multifunctional Reference Cells for Multistandard RFID Transponders, in: Microwave Theory and Techniques, IEEE Transactions on, in press, $2010 \mathrm{f}$.

Meyer, J., Herschmann, R., and Geck, B.: Antenna design for multistandard transponder ics, in: RFID-Systec 2010, June 2010a.

Meyer, J., Herschmann, R., and Geck, B.: A testbed for precision impedance measurements of planar symmetrical rfid antennas, in: EUMW 2010, 2010 b.

Smith, S. W.: The Scientist and Engineers's Guide to Digital Signal Processing, California Technical Publishing, 2006.

Vossiek, M., Roskosch, R., and Heide, P.: Precise 3-D Object Position Tracking using FMCW Radar, Proc. 29th European Microwave Conference, October 1999.

Wiebking, L.: Entwicklung eines zentimetergenauen mehrdimensionalen Nahbereichs-Navigationssystems, Duesseldorf, VDI Verlag GmbH, 2003. 